\section{Factores individuales y grupales que inciden en el intercambio de información entre implementadores de programas de intervención: una visión estructural}

\author{
Individual and group factors that impact the \\ exchange of information among intervention \\ programs: a structural view
}

Fatores individuais e de grupo que incidem na troca de informações entre implementadores de programas de intervenção: uma visão estrutural

\section{Resumen}

El intercambio de información entre los aplicadores de intervenciones en salud mental es esencial para garantizar la efectividad de la implementación de programas. El objetivo fue examinar cómo influyen factores individuales y grupales en el intercambio de información entre los implementadores de un programa de intervención psicosocial dirigido a víctimas de la guerra en Colombia. Se desarrolló un diseño exploratorio transversal. Fueron entrevistados 18 psicólogos, 22 trabajadores sociales, 10 promotores comunitarios y una enfermera. Se empleó el índice E-i y la visualización de vínculos interclase e intraclase. No existe tendencia homofílica en el intercambio de información entre las tres categorías profesionales. El empoderamiento predice las nominaciones recibidas en la red de solicitud de información $(\beta=0,296, p<0,006)$. Ser central en la red de preferencia es el principal predictor de la recepción de peticiones de información $(\beta=0,562, p<0,0001)$. Los profesionales empoderados y aquellos que intercambian información sobre el proceso de implementación son considerados informantes clave que pueden mejorar la efectividad del programa.

Intercambio de Información; Implementación de Plan de Salud; Servicios de Salud Mental; Conflictos Armados; Poblaciones Vulnerables
Ignacio Ramos-Vidal 1

Jorge Palacio 2

Alicia Uribe 3

Ilse C. Villamil 3

\author{
Correspondencia \\ I. Ramos-Vidal \\ Facultad de Psicología, Universidad de Sevilla. \\ Calle Camilo José Cela s/n, Sevilla 41018, España. \\ iramos5@us.es \\ 1 Facultad de Psicología, Universidad de Sevilla, Sevilla, España. \\ 2 Universidad del Norte, Barranquilla, Colombia. \\ 3 Universidad Pontificia Bolivariana, Montería, Colombia.
}




\section{Introducción}

El intercambio de información entre profesionales incide en la calidad de la atención psicosocial prestada, facilitando la (a) adopción de prácticas de intervención basadas en la evidencia empírica, (b) la difusión de innovaciones y (c) la resolución de incidencias durante la atención 1,2,3,4. Los problemas de comunicación afectan negativamente en la prestación de servicios, y la fluidez en el proceso de comunicación incide positivamente en la satisfacción de los pacientes y en la calidad de la atención 5 . Los factores que determinan el intercambio de información pueden ser individuales y grupales 6,7,8. Los profesionales que gozan de autonomía, competencia y percepción de control sobre el entorno de trabajo, intercambian información activamente 9,10. Estas dimensiones se asocian al empoderamiento en la ejecución de tareas.

El empoderamiento psicológico en el contexto profesional describe la medida en que los trabajadores adquieren dominio y pericia en relación a sus funciones, dicho proceso de adquisición de habilidades redunda en el desarrollo de competencias, haciendo posible incrementar la percepción de control sobre el entorno laboral 10,11. En atención primaria, son escasos los antecedentes que analizan la influencia del empoderamiento psicológico en el lugar de trabajo sobre el intercambio de información entre profesionales. El acceso a información médica incrementa el empoderamiento de profesionales y pacientes 12,13. Resulta pertinente analizar cómo influye el empoderamiento en el desarrollo de tareas y en la decisión de intercambiar información.

Hay una serie de variables externas que afectan en el intercambio de información entre profesionales. Los factores estructurales incluyen las características de las organizaciones que proporcionan la atención y variables grupales como el clima organizacional 14. La coordinación es más difícil cuanto más heterogéneos son los equipos. Los problemas de comunicación se derivan de la incompatibilidad entre diversidad y cohesión social 15,16. De allí que el clima organizacional sea un factor clave que describe el nivel de cohesión grupal e incide en la coordinación y el intercambio de información en los equipos 17 .

Los integrantes de los equipos de intervención tienden a intercambiar información con profesionales de la misma categoría. Esta tendencia se denomina homofilia y su estudio es clave para comprender la formación de redes 18 . En el contexto de las relaciones entre profesionales, la utilización del análisis de redes sociales (ARS) contribuye a diseñar y a gestionar de manera efectiva equipos de atención 19,20. El ARS evalúa cómo interaccionan entre sí distintos tipos de relaciones, es decir, cómo la posición que un actor ocupa en un tipo de relación (p.ej., intercambio de información) determina el rol de ese actor en otras relaciones (p.ej., adopción de buenas prácticas).

Los integrantes de equipos de atención intercambian información y reconocen como expertos a profesionales a los que les une una relación de amistad, y prefieren intercambiar información con colegas con los que han mantenido interacciones positivas previamente 21. Los profesionales con mayor número de conexiones en distintas relaciones se caracterizan por intercambiar información, derivar usuarios y adoptar buenas prácticas de intervención 22 . En sentido opuesto, ocupar posiciones periféricas dificulta el acceso a información y la adopción de buenas prácticas 23 .

Esta investigación examina cómo influye el empoderamiento psicológico, el clima organizacional y la posición ocupada en las redes de reconocimiento entre profesionales y de preferencia para trabajar, sobre el intercambio de información entre los implementadores de un programa de atención psicosocial a víctimas de la guerra en Colombia. Las hipótesis son: $\mathrm{H \# 1a} \mathrm{-} \mathrm{ocupar} \mathrm{posiciones} \mathrm{perifé-}$ ricas en la red de reconocimiento entre los implementadores se asocia a un bajo nivel de influencia en la red de intercambio de información, produciendo el efecto inverso la ocupación de posiciones centrales; $\mathrm{H} \# 1 \mathrm{~b}$ - ocupar posiciones periféricas en la red de preferencia para trabajar se asocia a un bajo nivel de influencia en la red de intercambio de información, produciendo el efecto inverso la ocupación de posiciones centrales; $\mathrm{H} \# 2$ - los profesionales intercambian información preferentemente con los integrantes de la misma categoría (homofilia); $\mathrm{H} \# 3$ - el empoderamiento se relaciona positivamente con las nominaciones recibidas y emitidas en la red de intercambio de información; $\mathrm{H} \# 4$ - el clima organizacional se asocia inversamente con las nominaciones recibidas y emitidas en la red de intercambio de información; $\mathrm{H} \# 5 \mathrm{a}$ - los actores con mayor centralidad de entrada (indegree) y de salida (outdegree) en la red de preferencia para trabajar son los más activos intercambiando infor- 
mación; $\mathrm{H} \# 5 \mathrm{~b}$ - los actores con mayor centralidad entrada (indegree) y de salida (outdegree) en la red de reconocimiento entre profesionales son los más activos intercambiando información.

\section{Materiales y métodos}

\section{Contexto de estudio}

El conflicto armado en Colombia ha generado cerca de un millón fallecidos y nueve millones de desplazados. En el Departamento de Córdoba, hay registradas más 400.000 víctimas de diferentes delitos en el Registro Único de Víctimas (Unidad para la Atención y la Reparación Integral a las Víctimas del Conflicto Armado. https://www.unidadvictimas.gov.co/es/registro-unico-de-victimas-ruv/37394, accedido el 19/Sep/2018). Según esta información, en torno al 20,9\% de la población residente la región ha sido víctima de algún acto delictivo vinculado al conflicto. El Programa de Atención Psicosocial y Salud Integral a Víctimas (PAPSIVI) fue diseñado para llevar a cabo un proceso de reparación de la salud mental de la población afectada por la violencia (Memoria técnica del Programa de Atención Psicosocial y Salud Integral a Víctimas (PAPSIVI). https://www.minsalud.gov.co/proteccionsocial/Paginas/Victimas_PAPSIVI.aspx, accedido el 10/Jul/2018). El programa proporciona atención psicosocial a nivel individual, familiar y comunitario a las víctimas. En 2017 fueron atendidas en Córdoba 8.803 víctimas, por un equipo compuesto por sesenta y siete personas (veintiséis psicólogos, veintiséis trabajadores sociales, trece facilitadores comunitarios, un enfermero y un médico).

\section{Diseño y muestreo}

La investigación es transversal y exploratoria. Esta investigación se adhiere a la Declaración STROBE para reportar estudios observacionales y transversales.

\section{Participantes}

Fueron entrevistados 51 de los 67 profesionales $(76,1 \%)$ que implementan el PAPSIVI en Córdoba. Los criterios de inclusión para participar en el estudio consistieron en que los profesionales estuvieran vinculados contractualmente a la Secretaría de Salud de la Gobernación de Córdoba (entidad responsable de ejecutar el programa en la región) y que llevaran al menos un mes trabajando como implementadores del PAPSIVI. Fueron excluidos de los análisis reportados en este trabajo los datos correspondientes a dos administrativas y una coordinadora operativa que realizan funciones de gestión no relacionadas con la implementación de las actividades del programa. De los 51 participantes 48 son mujeres (94,1\%). Participaron 18 psicólogos (35,3\%), 22 trabajadores sociales (43,1\%), 10 facilitadores comunitarios (19,6\%) y la enfermera de enlace (2\%). El análisis de redes se efectuó sobre 49 de los 51 profesionales, dado que la enfermera de enlace y un implementador no aparecieron en el censo proporcionado por los coordinadores del programa, por lo que no pudieron ser incluidos en el cuestionario socio-céntrico.

\section{Medidas estandarizadas}

Empleamos escalas estandarizadas para evaluar las dimensiones competencia ( 3 ítems) y autodeterminación (3 ítems) del empoderamiento psicológico de los participantes en el contexto de trabajo y las dimensiones inclusión (5 ítems), compromiso (4 ítems) y satisfacción (3 ítems) del clima organizacional 10,11. Los instrumentos están compuestos por afirmaciones que los entrevistados deben puntuar, en función de su grado de acuerdo con cada una de ellas, donde: 1 = nada de acuerdo y 4 = muy de acuerdo. Un ejemplo de ítem para evaluar la dimensión competencia del empoderamiento psicológico en el puesto de trabajo es la siguiente: "Tengo confianza en mi capacidad para hacer mi trabajo"; un ejemplo de afirmación para evaluar la dimensión inclusión del clima laboral es: "Todo el mundo participa en la toma de decisiones sobre acciones a seguir". Ambos instrumentos han sido previamente validados y presentan aceptables propiedades psicométricas como puede observarse en la Tabla 1. 
Tabla 1

Estadísticas descriptivas, fiabilidad de las escalas y matriz de correlaciones bivariadas entre las variables de estudio.

\begin{tabular}{|c|c|c|c|c|c|c|c|c|c|c|c|c|c|}
\hline Instrumento & $\mathbf{M}$ & DT & $a$ & 1 & 2 & 3 & 4 & 5 & 6 & 7 & 8 & 9 & 10 \\
\hline 1. Competencia (EP) & 11,20 & 0,95 & 0,64 & & & & & & & & & & \\
\hline 2. Autodeterminación(EP) & 10,20 & 1,40 & 0,75 & 0,49 * & & & & & & & & & \\
\hline 3. Inclusión (CO) & 14,90 & 1,30 & 0,61 & 0,08 & 0,11 & & & & & & & & \\
\hline 4. Compromiso (CO) & 14,40 & 2,30 & 0,66 & 0,26 & 0,18 & $0,33 * *$ & & & & & & & \\
\hline 5. Satisfacción (CO) & 16,10 & 2,70 & 0,87 & 0,20 & 0,22 & 0,33 ** & 0,50 * & & & & & & \\
\hline $\begin{array}{l}\text { 6. Indegrre } \\
\text { reconocimiento }\end{array}$ & 51,10 & 15,10 & $\mathrm{~N} / \mathrm{A}$ & $-0,11$ & $-0,05$ & $-0,16$ & $-0,40 * \star$ & $-0,20$ & & & & & \\
\hline $\begin{array}{l}\text { 7. Outdegree } \\
\text { reconocimiento }\end{array}$ & 51,10 & 23,60 & $\mathrm{~N} / \mathrm{A}$ & $-0,03$ & 0,11 & $-0,01$ & $-0,31 * \star$ & $-0,31 * *$ & 0,37 * & & & & \\
\hline 8. Indegree preferencia & 7,30 & 6,01 & $\mathrm{~N} / \mathrm{A}$ & 0,11 & $-0,01$ & $-0,14$ & $-0,19$ & $-0,27$ & 0,50 * & $0,28 * \star$ & & & \\
\hline 9. Outdegree preferencia & 7,30 & 7,01 & $\mathrm{~N} / \mathrm{A}$ & $0,28 * *$ & 0,34 * & 0,10 & $-0,04$ & $-0,01$ & $-0,06$ & $0,30 * \star$ & 0,23 & & \\
\hline $\begin{array}{l}\text { 10. Indegree intercambio } \\
\text { de información }\end{array}$ & 8,50 & 6,60 & $\mathrm{~N} / \mathrm{A}$ & $0,29 * *$ & 0,13 & $-0,05$ & $-0,21$ & $-0,23$ & 0,48 * & 0,24 & 0,71 * & 0,38 * & \\
\hline $\begin{array}{l}\text { 11. Outdegree } \\
\text { intercambio de } \\
\text { información }\end{array}$ & 8,50 & 6,30 & $\mathrm{~N} / \mathrm{A}$ & 0,10 & 0,31 * & 0,07 & $-0,13$ & $-0,10$ & $-0,06$ & 0,24 & $0,28 * *$ & 0,40 * & $0,29 * *$ \\
\hline
\end{tabular}

a: alfa de Cronbach; CO: clima organizacional; DT: desviación tipo; EP: empoderamiento psicológico; M: media; N/A: no aplica al no tratarse de escalas estandarizadas.

$* p<0,001$;

** $p<0,05$.

\section{Medidas relacionales}

Para analizar las relaciones entre profesionales se diseñó un cuestionario socio-céntrico (Material Suplementario. http://cadernos.ensp.fiocruz.br/static//arquivo/suppl-e00045420_7816.pdf). Cada una de las redes evaluadas (red de reconocimiento, de preferencia para trabajar y red de intercambio de información) se construyeron con las nominaciones que cada profesional hace al resto de profesionales que aparecen en el listado, que incluye los nombres de todos miembros del equipo de implementadores del PAPSIVI. Por ejemplo, para "generar" la red de reconocimiento, cada profesional debe marcar con una "X", en el listado que aparece en el Material Suplementario (http://cadernos.ensp. fiocruz.br/static//arquivo/suppl-e00045420_7816.pdf), a aquellos profesionales a los que reconoce por su nombre. Una vez que se disponen de las nominaciones que cada profesional efectúa al resto de implementadores, esta información es trasladada a una matriz de adyacencia en el software Ucinet (https://sites.google.com/site/ucinetsoftware/downloads). Cada matriz cuenta con el mismo número profesionales en las filas y en las columnas (en este caso, 49x49). Dos profesionales mantienen un "vínculo" si, por ejemplo, el profesional 1 reconoce al profesional 2. Por lo tanto, existen cuatro opciones posibles en cada relación diádica: (a) que el profesional 1 nomine al profesional 2, pero no a la inversa (relación unidireccional); (b) que el profesional 2 nomine al profesional 1, pero no a la inversa (relación unidireccional); (c) que los profesionales 1 y 2 se nominen mutuamente (relación bidireccional o recíproca); y (d) que no se dé ninguna nominación entre ambos (se rellenaría la casilla correspondiente con un 0). Considerando el tamaño de la red $(\mathrm{n}=49)$, para construir cada red es preciso cumplimentar las 2.352 relaciones diádicas posibles, excluyéndose las relaciones autorreflexivas.

Los indicadores de centralidad y cohesión se calcularon con Ucinet y la visualización de las redes se ejecutó con la aplicación NETDRAW, que está disponible en Ucinet. Para analizar el posicionamiento individual de cada profesional en cada red, se emplearon los indicadores grados de salida (outdegree) y grados de entrada (indegree). El indicador outdegree describe el número de nominaciones 
que un profesional ha realizado al resto de implementadores que aparecen en el listado, mientras que el valor de indegree muestra el número de nominaciones que un profesional ha recibido por parte del resto de profesionales. Por ejemplo, si un profesional reconoce a todos los profesionales que aparecen en el listado por su nombre, y al mismo tiempo es reconocido por todos los profesionales, presentará el valor máximo posible (48) en los indicadores indegree y outdegree de la red de reconocimiento.

Para caracterizar las redes en su conjunto, se utilizaron diferentes medidas de cohesión estructural que se describen a continuación. La densidad muestra a modo de porcentaje los vínculos que se dan en una red, en comparación con el número total de relaciones posibles. La transitividad examina la cohesión micro-local de las redes que se produce entre subconjuntos de tres actores (tríadas). Una tríada es transitiva cuando en un conjunto de tres actores se dan todas las relaciones posibles, es decir, que el profesional 1 reconozca al profesional 2, que el profesional 2 reconozca al profesional 3, y que el profesional 3 a su vez reconozca al profesional 1. Cuando esta medida se emplea para describir las propiedades de una red completa se expresa a modo de porcentaje, de tal manera que, si una red presenta una transitividad del $25 \%$, esto significa que 1 de cada 4 tríadas posibles es transitiva. La centralización muestra en qué medida las relaciones que se dan en una red se concentran en un subconjunto de actores. Las redes que presentan valores elevados de centralización (de grados de entrada o de salida) describen estructuras relacionales en las que una pequeña proporción de los sujetos que componen la red es la que participa en un mayor número de relaciones. El grado medio muestra las nominaciones que en promedio mantienen los integrantes de una red. Por ejemplo, en el caso de la red de reconocimiento que se observa en la Figura 1, el valor del grado medio es 24,55, lo que significa que, en promedio, cada profesional reconoce por su nombre a la mitad de los profesionales que trabajan en el programa PAPSIVI. El coeficiente de agrupabilidad indica en qué medida los actores de una red pueden formar parte de múltiples subconjuntos cohesivos de manera simultánea. Finalmente, para evaluar el nivel de homofilia o heterofilia de las redes en función del puesto de trabajo se empleó el índice E-i 24. Este parámetro analiza la proporción de vínculos que se mantienen con actores de la misma categoría (en este caso, del mismo perfil profesional, por ejemplo, relaciones entre psicólogos) y las relaciones que se mantienen con actores pertenecientes a otras categorías (por ejemplo, relaciones entre psicólogos y trabajadores sociales). Los valores del índice E-i oscilan entre -1, cuando los actores sólo establecen relaciones con actores de la misma categoría (homofilia pura) y 1 cuando todas las relaciones se mantienen con actores de distinta categoría (heterofilia pura).

Los análisis estadísticos se desarrollaron en SPSS (https://www.ibm.com/). De forma específica se analizaron las correlaciones bivariadas entre las variables en estudio y para testar las hipótesis propuestas se ejecutaron contrastes de medias a través de la prueba t de Student, y dos modelos de regresión jerárquica, siguiendo el procedimiento de pasos sucesivos (stepwise).

\section{Procedimiento}

Los coordinadores del programa fueron informados de los objetivos de la investigación y suscribieron un compromiso de confidencialidad al igual que los responsables del estudio. Los participantes firmaron un consentimiento informado. La aplicación del cuestionario duró entre 60-80 minutos. Miembros del equipo de investigación ofrecieron directrices detalladas a los participantes y estuvieron presentes durante el proceso de aplicación para resolver cualquier duda durante la cumplimentación del cuestionario.

\section{Estrategia de análisis}

Para testar la $\mathrm{H} \# 1$ a y la $\mathrm{H} \# 1 \mathrm{~b}$ identificamos la estructura centro-periferia 25 de las redes de reconocimiento y de preferencia para trabajar, al objeto de conocer si ocupar posiciones centrales o periféricas se relaciona con presentar un elevado nivel de indegree en la red de intercambio de información. Empleamos el índice E-i 24 para analizar la homofilia basada en el puesto $(\mathrm{H} \# 2)$, representando las relaciones intraclases e interclases en las tres redes evaluadas (Figuras 1a, 1b y 1c). Para testar las últimas tres hipótesis llevamos a cabo dos modelos de regresión jerárquica en los que la centralidad de grados de entrada (indegree) y de salida (outdegree) de la red de intercambio de información actúan 


\section{Figura 1}

Redes de intercambio de información entre los implementadores del Programa de Atención Psicosocial y Salud Integral a Víctimas (PAPSIVI) (n = 49).

1a) Intercambio de información

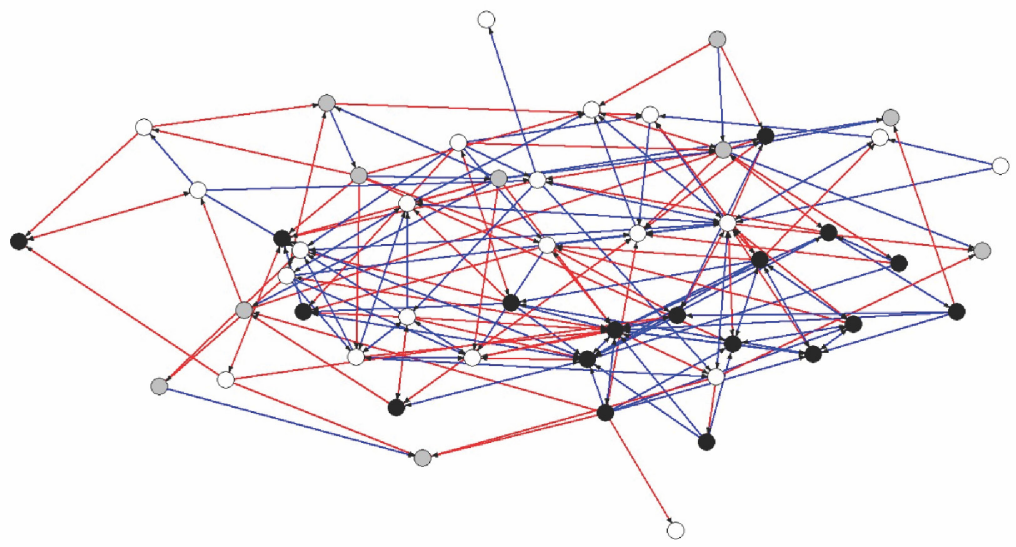

1b) Reconocimiento

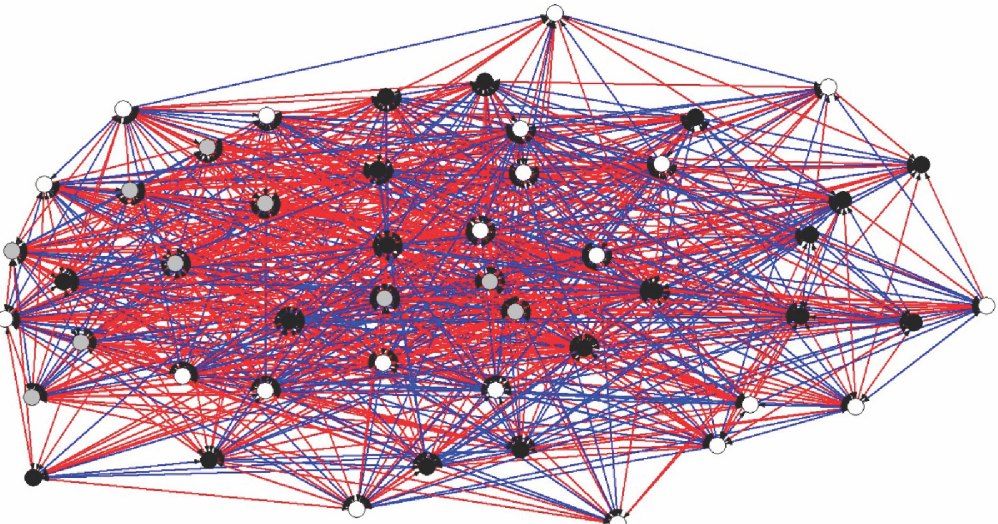

1c) Preferencia de colaboración

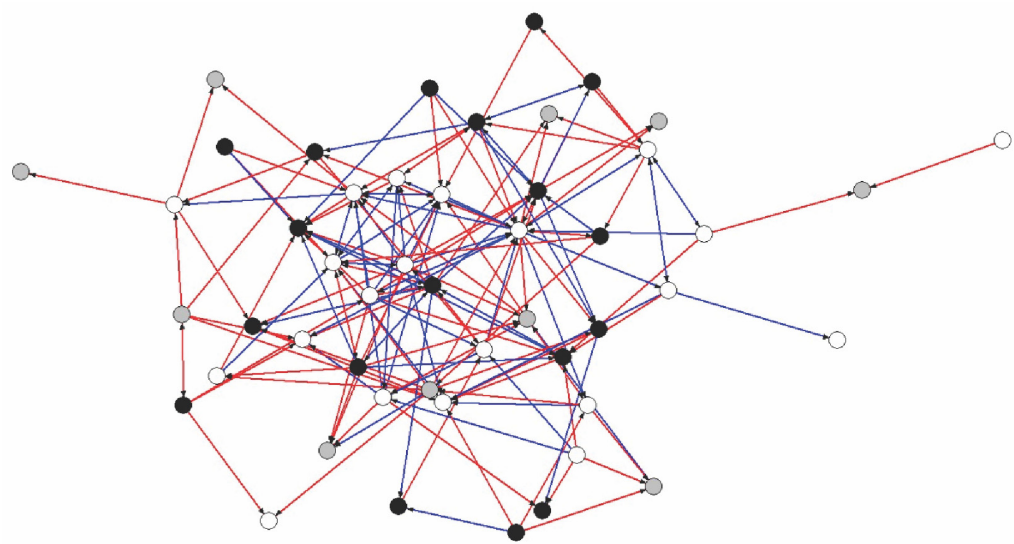

\section{Parámetros de cohesión}

Densidad (completa): $8,5 \%$

Densidad (ego): $19,13 \%$

Reciprocidad: $25 \%$

Transitividad: 7,04\%

Número de lazos: 200

Grado medio (Nm): 4,08

Centralización (entrada): 23,2\%

Centralización (salida): $23,2 \%$

Homofilia (índice E-i): -0,09

Coeficiente de agrupabilidad:

0,196

\section{Parámetros de cohesión}

Densidad (completa): 51,17\%

Densidad (ego): 59,71\%

Reciprocidad: $50 \%$

Transitividad: $36,64 \%$

Número de lazos: 1.203

Grado medio (Nm): 24,55

Centralización (entrada): $28,6 \%$

Centralización (salida): $49,8 \%$

Homofilia (índice E-i): 0,240

Coeficiente de agrupabilidad:

0,597

\begin{tabular}{|l|}
\hline \multicolumn{1}{|c|}{ Atributos } \\
\hline Color del nodo: categoria \\
Psicólogo/a: negro \\
Trabajador/a social: blanco \\
Promotor/a: gris \\
Color de la línea: relaciones \\
intergrupales e intragrupales \\
Roja: intergrupales \\
Azul: intragrupales \\
\end{tabular}

\begin{tabular}{|l|}
\hline \multicolumn{1}{|c|}{ Atributos } \\
\hline Color del nodo: categoria \\
Psicólogo/a: negro \\
Trabajador/a social: blanco \\
Promotor/a: gris \\
Color de la línea: relaciones \\
intergrupales e intragrupales \\
Roja: intergrupales \\
Azul: intragrupales \\
\end{tabular}

\section{Parámetros de cohesión}

Densidad (completa): 7,3\%

Densidad (ego): N.D.

Reciprocidad: $12,42 \%$

Transitividad: 7,11\%

Número de lazos: 172

Grado medio (Nm): 3,51

Centralización (entrada): 18,1\%

Centralización (salida): $24,4 \%$

Homofilia (índice E-i): 0,163

Coeficiente de agrupabilidad:

0,202

\begin{tabular}{|c|}
\hline \multicolumn{1}{|c|}{ Atributos } \\
\hline Color del nodo: categoria \\
Psicólogo/a: negro \\
Trabajador/a social: blanco \\
Promotor/a: gris \\
Color de la línea: relaciones \\
intergrupales e intragrupales \\
Roja: intergrupales \\
Azul: intragrupales \\
\end{tabular}

N.D.: no disponible. 
como dependientes, y empoderamiento $(\mathrm{H} \# 3)$, clima organizacional $(\mathrm{H} \# 4)$, y outdegree e indegree de las redes de preferencia para trabajar $(\mathrm{H} \# 5 \mathrm{a})$ y reconocimiento $(\mathrm{H} \# 5 \mathrm{~b})$ actúan respectivamente como independientes.

\section{Resultados}

En conjunto, la muestra lleva trabajando de media 12 meses en el programa (desviación tipo - DT $=12,41)$. Los promotores comunitarios conforman el grupo que lleva más tiempo trabajando en el programa, 24 meses en promedio, en comparación con los 6 meses de los psicólogos y los algo más de ocho meses de los trabajadores sociales $\left(\chi^{2}=13,100\right.$; g.l. $\left.=2 ; \mathrm{p}<0,001\right)$.

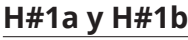

Se realizaron diferentes pruebas para identificar la estructura centro-periferia en la red de reconocimiento $(r=0,480)$ y en la red de preferencia para trabajar $(r=0,327)$, constatando la robustez de ambos modelos. En la red de reconocimiento se identifican 29 profesionales en el centro y 20 en la periferia. En la red de preferencia hay 16 profesionales centrales y 33 periféricos. Llevamos a cabo la prueba $t$ para muestras independientes para comprobar si la posición que los implementadores ocupan en ambas redes determina en el número de nominaciones recibidas (indegree) en la red de intercambio de información. Los actores situados en el centro de la red de reconocimiento presentan niveles superiores de indegree en la red de intercambio de información, en comparación con aquellos situados en la periferia $(t=2.776$; g.l. $=47 ; \mathrm{p}<0,008)$. La comparación de los grupos centrales y periféricos en función del indegree en la red de intercambio de información sustenta la $\mathrm{H \# 1a.} \mathrm{En} \mathrm{la} \mathrm{red}$ de preferencia, los implementadores que ocupan el centro también cuentan con mayor centralidad de entrada (indegree), en comparación con los periféricos ( $\mathrm{t}=4.623$; g.l. $=47 ; \mathrm{p}<0,0001)$, por lo que la $\mathrm{H} \# 1 \mathrm{~b}$ también se sustenta por los resultados.

\section{H\#2}

Se analizaron las interacciones entre las tres categorías profesionales en las redes de reconocimiento entre profesionales, preferencia para trabajar e intercambio de información. Calculamos el índice E-i 24 basado en la categoría profesional y, posteriormente, representamos las tres redes (Figura 1), identificando a través color del vínculo las relaciones intraclases (homofílicas) e interclases (heterofílicas).

Las redes de intercambio de información y de preferencia para trabajar presentan niveles moderados de densidad inferiores al $10 \%$ en ambos casos, a diferencia de la red de reconocimiento que presenta una densidad comparativamente elevada por encima del 50\%. Estos valores, al igual que los que reflejan el coeficiente de agrupabilidad y el porcentaje de tríadas transitivas, muestran niveles de cohesión estructural bajos en las redes de intercambio de información y de preferencia para trabajar, y elevado en la red de reconocimiento entre profesionales. En el caso de la red de intercambio de información, es frecuente que las redes que denotan este tipo de interacción profesional presenten valores moderados, en la medida en que las oportunidades para que dos implementadores intercambien información profesional dependen en cierta medida de que formen parte del mismo equipo de intervención o que presten atención en los mismos contextos comunitarios. Sin embargo, considerando este condicionante, sería aconsejable implementar mecanismos para aumentar el intercambio de información relacionada con el proceso de intervención, dado que la evidencia empírica sugiere que esta actividad contribuye positivamente a mejorar la efectividad de las intervenciones 1,3,4,6.

El índice E-i de la red de intercambio de información arroja un valor negativo próximo a cero $(\mathrm{E}-\mathrm{i}=$ $-0,009)$ que muestra que, para esta relación, la categoría profesional no influye en el intercambio de información. En la red de reconocimiento se observa una tendencia heterofílica moderada (E-i = 0,240 ), por su parte la red de preferencia muestra una tendencia heterofílica igualmente moderada $(E-i=0,163)$. La representación de las relaciones intraclase e interclase evidencia que no existe una tendencia homofílica. La segunda hipótesis queda rechazada al no identificarse dinámica de relaciones homofílicas entre los profesionales de cada perfil profesional. 


\section{H\#3, H\#4, H\#5a y H\#5b}

Las hipótesis finales son testadas de manera conjunta mediante dos modelos de regresión jerárquicos. Mediante sendos modelos se pretende conocer los efectos del empoderamiento $(\mathrm{H} \# 3)$, el clima $(\mathrm{H} \# 4)$ y la posición ocupada en la red de preferencia para trabajar $(\mathrm{H} \# 5 \mathrm{a})$ y de reconocimiento $(\mathrm{H} \# 5 \mathrm{~b})$, sobre el valor de indegree (modelo 1) y de outdegree (modelo 2) en la red de intercambio de información. En la Tabla 1 son mostrados los estadísticos descriptivos, la fiabilidad de las escalas y la matriz de correlaciones entre las variables analizadas. En la Tabla 2 aparecen los coeficientes del primer modelo de regresión y en la Tabla 3 se describen los coeficientes del segundo modelo de regresión.

No se observan relaciones de covarianza significativas entre el empoderamiento y el clima organizacional. Las dimensiones competencia y autodeterminación (empoderamiento) no correlacionan con la centralidad de salida (outdegree) de la red de preferencia y con la de entrada (indegree) en la red de intercambio de información. Las dimensiones compromiso y satisfacción del clima organizacional

Tabla 2

Coeficientes de regresión prediciendo la centralidad de entrada (indegree) en la red de solicitud de información (modelo 1).

\begin{tabular}{|c|c|c|c|c|c|c|c|}
\hline Variables dependientes y paso & B & $\beta$ & $\mathbf{t}$ & Valor de p & $\mathrm{IC95 \%}$ & Tolerancia & FIV \\
\hline \multicolumn{8}{|l|}{ Paso 1. Empoderamiento psicológico } \\
\hline Competencia & 2,003 & 0,296 & 2,891 & 0,006 & 0,$604 ; 3,401$ & 0,859 & 1,165 \\
\hline \multicolumn{8}{|c|}{ Paso 2. Dimensiones del clima organizacional } \\
\hline Inclusión & $-0,279$ & $-0,097$ & $-0,097$ & 0,440 & $-1,002 ; 0,443$ & 0,584 & 1,713 \\
\hline Compromiso & 0,466 & 0,098 & 0,946 & 0,350 & $-0,529 ; 1,460$ & 0,845 & 1,184 \\
\hline Satisfacción & $-0,209$ & $-0,088$ & $-0,752$ & 0,456 & $-0,769 ; 0,351$ & 0,656 & 1,524 \\
\hline \multicolumn{8}{|c|}{ Paso 3. Grados de entrada (indegree) } \\
\hline Red de reconocimiento & 0,088 & 0,203 & 1,701 & 0,097 & $-0,017 ; 0,193$ & 0,631 & 1,585 \\
\hline Red de preferencia & 0,609 & 0,562 & 4,840 & 0,0001 & 0,$355 ; 0,863$ & 0,667 & 1,498 \\
\hline
\end{tabular}

ß: coeficiente de regresión estandarizado; B: coeficiente de regresión $\beta$ no estandarizado; FIV: factor de inflación de la varianza; IC95\%: intervalo de 95\% de confianza.

Tabla 3

Coeficientes de regresión prediciendo la centralidad de salida (outdegree) en la red de solicitud de información (modelo 2).

\begin{tabular}{|c|c|c|c|c|c|c|c|}
\hline Variables dependientes y paso & B & $\beta$ & $\mathbf{t}$ & $\mathbf{p}$ & IC95\% & Tolerancia & FIV \\
\hline \multicolumn{8}{|l|}{ Paso 1. Empoderamiento psicológico } \\
\hline Autodeterminación & 1,348 & 0,298 & 2,048 & 0,047 & 0,$018 ; 2,679$ & 0,788 & 1,269 \\
\hline \multicolumn{8}{|c|}{ Paso 2. Dimensiones del clima organizacional } \\
\hline Inclusión & $-0,811$ & $-0,291$ & $-1,699$ & 0,097 & $-1,775 ; 0,154$ & 0,569 & 1,758 \\
\hline Compromiso & 0,628 & 0,136 & 0,959 & 0,343 & $-0,695 ; 1,951$ & 0,827 & 1,209 \\
\hline Satisfacción & $-0,074$ & $-0,032$ & $-0,202$ & 0,841 & $-0,811 ; 0,664$ & 0,656 & 1,525 \\
\hline \multicolumn{8}{|l|}{ Paso 3. Grados de salida (outdegree) } \\
\hline Red de preferencia & 0,157 & 0,172 & 1,154 & 0,255 & $-0,118 ; 0,433$ & 0,748 & 1,336 \\
\hline \multicolumn{8}{|l|}{ Paso 4. Grados de entrada (indegree) } \\
\hline Red de reconocimiento & $-0,134$ & $-0,318$ & $-1,901$ & 0,065 & $-0,277 ; 0,008$ & 0,593 & 1,686 \\
\hline Red de preferencia & 0,376 & 0,358 & 2,193 & 0,034 & 0,$030 ; 0,722$ & 0,624 & 1,603 \\
\hline
\end{tabular}

ß: coeficiente de regresión estandarizado; B: coeficiente de regresión $\beta$ no estandarizado; FIV: factor de inflación de la varianza; IC95\%: intervalo de 95\% de confianza. 
correlacionan significativamente en sentido inverso con la centralidad de salida (outdegree) de la red de reconocimiento. El resumen del primer modelo de regresión jerárquica muestra que en conjunto las variables introducidas explican el $57 \%$ de la varianza de la dependiente $\left(\Delta \mathrm{R}^{2}=0,577\right.$; media cuadrática $-\mathrm{MC}=216,739 ; \mathrm{p}<0,0001$; Estadístico de durbin-Watson $-\mathrm{DW}=2,104)$. El segundo modelo explica el $21 \%$ de la varianza de la centralidad de salida (outdegree) en la red de intercambio de información $\left(\Delta \mathrm{R}^{2}=0,218 ; \mathrm{MC}=92,212 ; \mathrm{p}<0,016 ; \mathrm{DW}=2,157\right)$.

La competencia relativa al empoderamiento en el contexto de trabajo explica una proporción considerable de la varianza ( $\beta=0,296$; $<<0,006$; IC95\%: 0,604; 3,401) de indegree en la red de intercambio de información (modelo 1). La autodeterminación relativa al empoderamiento en el contexto de trabajo explica la variabilidad de outdegree ( $\beta=0,298$; $p<0,047$; IC95\%: 0,018; 2,679) en la red de intercambio de información (modelo 2). El efecto de ambas dimensiones del empoderamiento en outdegree e indegree en la red de intercambio de información soporta la tercera hipótesis.

Las dimensiones del clima organizacional (inclusión, compromiso y satisfacción) covarían inversamente con los parámetros outdegree e indegree de las tres redes evaluadas. Esta covariación negativa es estadísticamente significativa entre la dimensión compromiso y la centralidad de entrada y de salida en la red de reconocimiento, y entre la dimensión satisfacción y la centralidad de salida en esa misma red. Cuando introducimos estas variables en sendas ecuaciones de regresión, ninguna explica significativamente la varianza de la dependiente. Estos resultados no sustentan la cuarta hipótesis.

$\mathrm{La} \mathrm{H} \# 5 \mathrm{a}$ y la $\mathrm{H} \# 5 \mathrm{~b}$ se propusieron para conocer en qué medida ser central, es decir, presentar valores elevados en los indicadores indegree y outdegree en la red de reconocimiento y de preferencia para trabajar, se asocia con ser un profesional que presenta valores elevados en outdegree e indegree en la red de intercambio de información. La variable con mayor capacidad para predecir la centralidad de entrada (modelo 1) y de salida (modelo 2) en la red de intercambio de información es el indegree en la red de preferencia para trabajar. El efecto de este indicador en la red de preferencia es superior en la centralidad de entrada (indegree) en la red de intercambio de información ( $\beta=0,562 ; \mathrm{t}=4,840$; $\mathrm{p}<0,0001)$, en comparación con la centralidad de salida $(\beta=0,358 ; \mathrm{t}=2,193 ; \mathrm{p}<0,034)$. Por tanto, ni la centralidad de salida en la red de preferencia para trabajar, ni la centralidad de entrada y de salida en la red de reconocimiento, condicionan la varianza de outdegree e indegree en la red de intercambio de información. Estos resultados ofrecen apoyo pleno a la $\mathrm{H} \# 5$ a en la medida en que el parámetro indegree de la red de preferencia para trabajar contribuye de forma significativa $(\beta=0,562 ; \mathrm{t}=4,840$; $\mathrm{p}<0,0001$ ) a explicar la varianza de la dependiente del primer modelo de regresión (indegree red de intercambio de información), y también contribuye a explicar la varianza de la variable dependiente en el segundo modelo de regresión $(\beta=0,358 ; \mathrm{t}=2,193 ; \mathrm{p}<0,034)$ en el que variable la dependiente es el indicador outdegree de la red de intercambio de información. En sentido inverso, los resultados no permiten confirmar la $\mathrm{H} \# 5 \mathrm{~b}$, dado que el indicador indegree de la red de reconocimiento no índice de forma estadísticamente significativa en la varianza de la variable dependiente de los modelos de regresión $1(\beta=0,203 ; \mathrm{t}=1,701 ; \mathrm{p}<0,097)$ y $2(\beta=-0,318 ; \mathrm{t}=-1,901 ; \mathrm{p}<0,065)$.

\section{Discusión}

Este artículo analiza la influencia del empoderamiento psicológico en el contexto de trabajo, del clima organizacional y de la posición ocupada en la red de reconocimiento y de preferencia, en los valores de outdegree e indegree en la red de intercambio información entre los implementadores del PAPSIVI. La literatura muestra que el intercambio de información repercute positivamente en la calidad del servicio prestado por los profesionales 4,6,7,8,26. Los profesionales suelen intercambiar información con colegas de la misma categoría, siendo predominantes las relaciones homofílicas 14,18 . Sin embargo en este estudio no se observa esa tendencia. En las redes de reconocimiento, preferencia para trabajar e intercambio de información evaluadas no se aprecia preferencia a establecer relaciones dentro de la misma categoría, incluso en las redes de reconocimiento y de preferencia para colaborar se identificó una tendencia heterofílica moderada. Las características organizativas del programa podrían explicar, al menos parcialmente, este resultado. Los equipos están conformados por un profesional de cada categoría, esto hace que la comunicación intragrupal se vea facilitada por la proximidad dentro del equipo, mientras que la comunicación intergrupal queda limitada por la distancia entre los equipos 
que prestan servicios en diferentes municipios. Estos factores determinan en cierta medida las posibilidades reales que los profesionales tienen para interactuar con miembros de otros equipos, y por lo tanto también afectan a la composición de las redes y a sus propiedades estructurales. Por otro lado, la conformación multidisciplinar de los equipos, que incluye, al menos, un psicólogo, un trabajador social y un promotor comunitario, facilita la interacción entre profesionales de diferente perfil, algo que a su vez puede incidir en los niveles de heterofilia detectados en las redes de reconocimiento y de preferencia para colaborar.

Otro hecho que puede explicar esta tendencia heterofílica es que cada profesional actúa preferentemente en un nivel de intervención (individual, familiar y comunitario). Los psicólogos desarrollan intervenciones individuales, fundamentalmente psicoterapia y en menor medida terapia con la unidad familiar. Los trabajadores sociales intervienen prioritariamente a nivel comunitario, aunque también desarrollan actividades con las familias. Mientras que los promotores comunitarios no intervienen a ninguno de estos niveles, pero actúan de nexo entre los otros dos perfiles profesionales y los usuarios del programa. Estas intersecciones entre los niveles de intervención facilitan el que profesionales de diferente perfil profesional colaboren durante el proceso de intervención psicosocial.

Dado que los equipos de intervención suelen estar asignados a municipios distantes entre sí, los miembros de los equipos tienen mayores oportunidades para intercambiar experiencias con implementadores del mismo equipo (factor que podría estar reforzando la heterofilia en base al perfil profesional), que con integrantes de distintos equipos (lo que fortalecería la homofilia en función del perfil profesional). A través de información interna proporcionada por los responsables del programa, se puede determinar que cada equipo brinda atención en comunidades adscritas a diferentes municipios, factor que puede propiciar la interacción dentro de los equipos, dificultando al mismo tiempo la comunicación con equipos asignados a otros municipios. De igual modo, considerando la amplia cobertura geográfica del programa que presta atención en los 30 municipios, en los que se divide administrativamente el Departamento de Córdoba, se puede afirmar que esto puede estar condicionando las interacciones entre los implementadores del programa.

La evidencia empírica apunta a que la dispersión geográfica dificulta las tareas de coordinación y gestión operativa de los programas de intervención 27 . Sería recomendable activar canales de comunicación para incrementar el flujo de información entre los implementadores del mismo perfil profesional, sistematizar lecciones aprendidas y difundir prácticas de intervención basadas en la evidencia. Las reuniones de coordinación son el contexto idóneo para intercambiar experiencias, esto posiblemente reforzaría conjuntamente la homofilia basada en el perfil profesional y la heterofilia en función del equipo de intervención 7,9,14.

Finalmente, el empoderamiento es un factor clave en el intercambio de información. La competencia relativa a la ejecución de tareas es una variable relevante para recibir peticiones de información. Los profesionales más competentes son influyentes y son recursos activos para obtener información por parte de otros profesionales. Aunque esta investigación se centra en examinar cómo influye el empoderamiento en el puesto de trabajo en la centralidad de entrada y de salida en la red de intercambio de información, posiblemente se esté dando un efecto bidireccional, es decir, que el acceso a información incrementa el empoderamiento. A continuación, son descritas las principales conclusiones de la investigación.

\section{Conclusión}

El empoderamiento es un proceso relacional y tener acceso a fuentes de información diversas incrementa el control sobre el entorno y la pericia de los implementadores de programas ${ }^{20}$. La solicitud de información es una estrategia adaptativa para responder a las demandas de la intervención. Los profesionales autónomos solicitan información a otros compañeros para prestar atención efectiva. Esto sugiere que, en la implementación del programa, solicitar información y recibir peticiones de información, son signos de empoderamiento en el contexto laboral. Identificar a los profesionales activos en el intercambio de información es relevante porque estos profesionales pueden actuar como promotores de cambios estructurales, difusores de buenas prácticas y adoptadores tempranos de nuevos protocolos de intervención 8,14,20,21. 
A nivel colectivo, se planteó que el clima de los equipos influye en sentido negativo sobre el intercambio de información. La cohesión grupal se relaciona inversamente con establecer relaciones con grupos diversos 15,16,18. Esto se explica debido a que los sujetos que perciben un buen clima, en cierta medida pueden ver satisfechas sus expectativas en términos relacionales, algo que desincentiva entablar contactos con miembros de otros equipos. Aunque el clima se relaciona con el rendimiento laboral, en el contexto del PAPSIVI, es aconsejable promover relaciones entre equipos y entre los diferentes perfiles profesionales que implementan el programa. Esto permitiría simultáneamente incrementar las relaciones entre los implementadores, intercambiar experiencias y generar sentido de pertenencia hacia las metas del programa.

\section{Colaboradores}

I. Ramos-Vidal participó del diseño de la investigación, procesamiento de los datos, análisis de datos, gestión del proyecto de investigación, escritura del primero borrador y revisión de la versión final. J. Palacio colaboró en el procesamiento y análisis de los datos, escritura del primero borrador y revisión de la versión final. A. Uribe y I. C. Villamil colaboraron en el procesamiento de los datos, gestión del proyecto de investigación y revisión de la versión final.

\section{Informaciones adicionales}

ORCID: Ignacio Ramos-Vidal (0000-0002-18400761); Jorge Palacio (0000-0001-6971-7067); Alicia Uribe (0000-0002-1244-8908); Ilse C. Villamil (0000-0001-5866-0544).

\section{Agradecimientos}

Al Centro para la Innovación en Desarrollo y la Investigación, Universidad Pontificia Bolivariana.

\section{Referencias}

1. Menachemi N, Rahurkar S, Harle CA, Vest JR. The benefits of health information exchange: an updated systematic review. J Am Med Inform Assoc 2018; 25:1259-65.

2. Coleman JS, Katz E, Menzel H. Medical innovation: a diffusion study. Indianapolis: BobbsMerrill; 2002.

3. Davoody N, Koch S, Krakau I, Hägglund M. Accessing and sharing health information for post-discharge stroke care through a national health information exchange platform: a case study. BMC Med Inform Decis Mak 2019; 19:95.

4. Fontaine P, Ross SP, Zink T, Schilling L. Systematic review of health information exchange in primary care practices. J Am Board Fam Med 2010; 23:655-70.

5. Zwarenstein M, Goldman J, Reeves S. Interprofessional collaboration: effects of practicebased interventions on professional practice and healthcare outcomes. Cochrane Database Syst Rev 2009; (3):CD000072.

6. Vest JR, Zhao H, Jasperson J, Gamm LD, Ohsfeldt RL. Factors motivating and affecting health information exchange usage. J Am Med Inform Assoc 2011; 18:143-9.

7. Edwards M, Mynfanwy D, Edwards A. What are the external influences on information exchange and shared decision-making in healthcare consultations: a meta-synthesis of the literature. Patient Educ Couns 2009; 75:37-52.

8. Fattore G, Frosini F, Salvatore D, Tozzi V. Social network analysis in primary care: the impact of interactions on prescribing behaviour. Health Policy 2009; 92:141-8.

9. Morgan PI, Ogbonna E. Subcultural dynamics in transformation: a multi-perspective study of healthcare professionals. Hum Relat 2008; 61:39-65.

10. Zimmerman MA. Psychological empowerment: issues and illustrations. Am J Commun Psychol 1995; 23:581-99. 
11. Spreitzer GM. Psychological empowerment in the workplace: dimensions, measurement and validation. Acad Manag J 1995; 38:1441-65.

12. Mesko B, Györffy Z. The rise of the empowered physician in the digital health era. J Med Internet Res 2019; 21:e12490.

13. Fox NJ, Ward KJ, O'Rourke AJ. The 'expert patient': empowerment or medical dominance? The case of weight loss, pharmaceutical drugs and the internet. Soc Sci Med 2005; 60:1299309.

14. West E, Barron DN, Dowsett JN, Newton JN. Hierarchies and cliques in the social networks of health care professionals: implications for the design of dissemination strategies. Soc Sci Med 1999; 48:633-46.

15. Neal ZP, Watling-Neal J. The (in)compatibility of diversity and sense of community. Am J Commun Psychol 2014; 53:1-12.

16. Pagani C. Diversity and social cohesion. Intercultural Education 2014; 25:300-11.

17. Chen CJ, Huang JW. How organizational climate and structure affect knowledge management - the social interaction perspective. Int J Inf Manage 2007; 27:104-18.

18. McPherson M, Smith-Lovin L, Cook JM. Birds of a feather: homophily in social networks. Annu Rev Sociol 2001; 27:415-44.

19. Luke D, Harris JK. Network analysis in public health: history, methods and applications. Annu Rev Public Health 2007; 28:69-93.

20. Meltzer D, Chung J, Khalili P, Marlow E, Arora V, Schumock G, Burt R. Exploring the use of social network methods in designing healthcare quality improvement teams. Soc Sci Med 2010; 71:1119-30.
21. Yousefi-Nooraie R, Dobbins M, Marin A. Social and organizational factors affecting implementation of evidence-informed practice in a public health department in Ontario: a network modeling approach. Implement Sci 2014; 9:29.

22. Shearer JC, Dion M, Lavis JN. Exchanging and using research evidence in health policy networks: a statistical network analysis. Implement Sci 2014; 9:126.

23. Gainforth HL, Latimer-Cheung AE, Athanasopoulos P, Moore S, Martin-Ginis KA. The role of interpersonal communication in the process of knowledge mobilization within a community-based organization: a network analysis. Implement Sci 2014; 9:59.

24. Krackhardt D, Stern RN. Informal networks and organizational crises: an experimental simulation. Soc Psychol Q 1988; 51:123-40.

25. Borgatti SP, Everett MG. Models of core/ periphery structures. Soc Networks 1999; 21:375-95.

26. Isba R, Woolf K, Hanneman R. Social network analysis in medical education. Med Educ 2017; 51:81-8.

27. Long JC, Cunningham FC, Carswell P, Braithwaite J. Patterns of collaboration in complex networks: the example of a translational research network. BMC Health Serv Res 2014; $14: 225$. 


\section{Abstract}

The exchange of information between appliers of mental health interventions is essential to guarantee effective program implementation. The study aimed to examine how individual and group factors influence the exchange of information among the implementers of a psychosocial intervention program targeted to victims of the war in Colombia. A cross-sectional exploratory design was developed. Interviews were held with 18 psychologists, 22 social workers, 10 community promoters, and a nurse. The study used the E-i index and the visualization of interclass and intraclass links. There is no homophilic tendency in the exchange of information among the three professional categories. Empowerment predicts the nominations received in the request for information network $(\beta=0.296, p<0.006)$. To be central in the preference network is the principal predictor of receiving requests for information $(\beta=0.562, p<0.0001)$. Empowered professionals and those who exchange information on the implementation process are considered key informants that can improve the program's effectiveness.

Information Sharing; Health Plan

Implementation; Mental Health Services; Armed Conflicts; Vulnerable Populations

\section{Resumo}

A troca de informações entre os profissionais envolvidos nas intervenções de saúde mental é fundamental para garantir a efetividade da implementação de programas. O objetivo aqui era analisar a maneira como fatores individuais e de grupo influenciam a troca de informações entre os implementadores de um programa de intervenção psicossocial, dirigido a vítimas da guerra na Colômbia. Foi desenvolvido um desenho de estudo exploratório transversal. Foram entrevistados 18 psicólogos, 22 trabalhadores sociais, 10 agentes comunitários e uma enfermeira. Foram utilizados o indice E-i e a visualização de vínculos interclasse e intraclasse. Não existe tendência homofílica na troca de informações entre as três categorias profissionais. O empoderamento prevê as denominações recebidas na rede de solicitação de informação $(\beta=0,296, p<0,006)$. Ser central na rede de preferência é o principal preditor da recepção de pedidos de informação $(\beta=0,562, p<0,0001)$. Os profissionais empoderados e aqueles que trocam informações sobre o processo de implementação são considerados informantes chaves que podem melhorar a efetividade do programa.

Intercâmbio de Informação; Implementação de Plano de Saúde; Serviços Saúde Mental; Conflitos Armados; Populações Vulneráveis
Recibido el 10/Mar/2020

Versión final presentada el 11/Sep/2020

Aprobado el 09/Oct/2020 